\title{
Beamforming for the Underlay Cognitive MISO Interference Channel via UL-DL Duality
}

\author{
Francesco Negro*, Irfan Ghauri ${ }^{\dagger}$, Dirk T.M. Slock* \\ ${ }^{\dagger}$ Infineon Technologies France SAS, GAIA, 2600 Route des Crêtes, 06560 Sophia Antipolis Cedex, France \\ Email: irfan.ghauri@infineon.com \\ *Mobile Communications Department, EURECOM, 2229 Route des Crêtes, BP 193, 06904 Sophia Antipolis Cedex, France \\ Email: francesco.negro@eurecom.fr, dirk.slock@eurecom.fr
}

\begin{abstract}
SINR duality is shown in a multi-input singleoutput (MISO) downlink (DL) interference channel (IFC) and its uplink (UL) single-input multi-output (SIMO) dual with linear transmit (Tx) beamformers (BF). While UL-DL duality under the sum power constraint is well-established between the broadcast channel (BC) for the SINR balancing (max min SINR) beamforming problem and its (easier to solve) UL multipleaccess (MAC) dual channel, such duality does not at first seem relevant for the IFC. We show that SINR duality under the sum power constraint nevertheless holds in the MISO IFC leading to BF design through similar considerations as the BC-MAC case. We next impose further per-Tx power constraints meaningful for the IFC structure and show continued existence of SINR duality in the MISO IFC and the corresponding UL SIMO dual channel, but this time with an uncertain UL noise scaling. The beamformers, $T x$ powers and noise variances are found through an iterative algorithm. We extend the solution to a cognitive radio network where this MISO IFC is co-deployed in a underlay fashion with a primary network with interference power constraints on primary single antenna receivers.
\end{abstract}

\section{INTRODUCTION}

Widespread deployment of wireless communications in recent years has made radio spectrum exceedingly crowded. This realization has lead the regulatory bodies to encourage usage of available spectrum in more efficient ways. Cognitive Radio (CR) is a set of techniques permitting efficient spectrum use. $\mathrm{CR}$ allows spectrum reuse between legacy (primary) networks (PN) and secondary (possibly opportunistic) networks ( $\mathrm{SN}$ ) as long as the latter do not hamper the formers' communications in an overly adverse fashion. For instance, underlay deployment of a CR is said to be in place when a given concerted level of interference from secondary Tx may be tolerated at the primary user (PU).

Underlay CR using multiple antennas has recently come under intense focus since in such systems spatial dimensions can be exploited to shape interference towards primary users. A general framework is presented in [1]. Much of the work in underlay $\mathrm{CR}$ systems has been in the context of secondary broadcast (BC) networks coexisting with primary users. This is essentially due to relatively good understanding of $\mathrm{BC}$ beamforming and power allocation problem acquired in recent years [2] [3] based on the principle of UL-DL duality. Using this duality, the BF designed in the virtual (dual) uplink mode can be used in the actual downlink problem to achieve the same SINR values by choosing appropriate downlink power allocations. The design of secondary $\mathrm{Tx}$ beamformers under primary interference constraints has, for example, been studied in [4] with the objective of SINR balancing in the SN. The role of UL-DL duality principle remains instrumental in the solution of this problem.

In this paper we focus on a $\mathrm{SN}$ that is no longer a $\mathrm{BC}$ but a MISO IFC. There is one fundamental difference between linear $\mathrm{BF}$ design and power allocation problems in $\mathrm{BC}$ and IFC, namely there are individual power constraints in the latter as opposed to a total power constraint in the former. Nevertheless, we argue that minimizing total Tx power in the IFC still makes sense from green wireless point of view and thus still makes a valid optimization problem.

We initially focus on the duality regime in the MISO-SIMO IFC in order to identify if any structure similar to UL-DL duality of the BC exists in this case. Zero-forcing duality and the more specific interference alignment duality are known to hold in the $K$-user IFC [5] [6]. To the best of our knowledge, no other results proving any other kind of duality have been established for the IFC. In this work, we show that UL-DL SINR duality holds for the MISO IFC. We also show that interestingly, the mechanics of this duality are quite similar to the UL-DL duality in the $\mathrm{BC}$ setting. This observation allows beamformer design in the MISO IFC using the same techniques as the ones well-known in the $\mathrm{BC}$ channel.

Beamformer design in presence of primary interference constraints is addressed. The primary users can be seen as a pair of receiver and virtual primary Tx, thus a supplementary interference link causing zero interference to the SN. The extended problem can be readily solved using the new result on UL-DL IFC duality obtained in this paper.

\section{General IFC Signal Model}

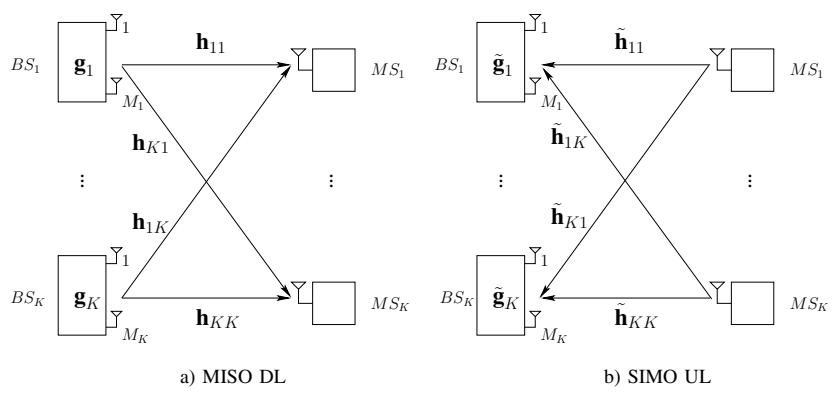

Fig. 1: System Model 
Fig. 1 depicts a $K$-user MISO IFC with $K$ transmitterreceiver pairs. The $k$-th Base Station (BS) is equipped with $M_{k}$ transmitter antennas and $k$-th mobile station (MS) is a single antenna node. The $k$-th transmitter generates interference at all $l \neq k$ receivers. Assuming the communication channel to be frequency-flat, the received signal $y_{k}$ at the $k$-th receiver, can be represented as

$$
y_{k}=\mathbf{h}_{k k} \mathbf{x}_{k}+\sum_{\substack{l=1 \\ l \neq k}}^{K} \mathbf{h}_{k l} \mathbf{x}_{l}+n_{k}
$$

where $\mathbf{h}_{k l} \in \mathbb{C}^{1 \times M_{l}}$ represents the channel vector between the $l$-th transmitter and $k$-th receiver, $\mathbf{x}_{k}$ is the $\mathbb{C}^{M_{k} \times 1}$ transmit signal vector of the $k$-th transmitter and $n_{k}$ represents (temporally white) AWGN with zero mean and variance $\sigma_{k}^{2}$. Each entry of the channel matrix is a complex random variable drawn from a continuous distribution. It is assumed that each transmitter has complete knowledge of all channel vectors.

We denote by $\mathbf{g}_{k}$, the $\mathbb{C}^{M_{k} \times 1}$ precoding matrix of the $k$-th transmitter. Thus $\mathbf{x}_{k}=\mathbf{g}_{k} s_{k}$, where $s_{k}$ represents the independent symbol for the $k$-th user pair. We assume $s_{k}$ to have a temporally white Gaussian distribution with zero mean and unit variance. In the SIMO UL channel the $k$-th BS applies a receiver $\tilde{\mathbf{f}}_{k}$ to suppress interference and retrieve its desired symbol. The output of such a receive filter is then given by

$$
\tilde{r}_{k}=\tilde{\mathbf{f}}_{k} \tilde{\mathbf{h}}_{k k} \tilde{s}_{k}+\sum_{\substack{l=1 \\ l \neq k}}^{K} \tilde{\mathbf{f}}_{k} \tilde{\mathbf{h}}_{k l} \tilde{s}_{l}+\tilde{\mathbf{f}}_{k} \tilde{n}_{k}
$$

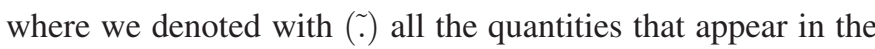
UL in order to differentiate with the same quantities in the DL.

\section{UL-DL DUALITY IN MISO/SIMO INTERFERENCE Channel Under Sum Power Constraint}

In this section we will derive UL-DL duality for a MISO IFC under a total power constraints To simplify the following analysis henceforth we assume that each receiver is characterized by the same noise variances, so $\sigma_{k}^{2}=\sigma^{2}, \forall k$. The received signal for the MISO DL IFC at the $k$-th mobile station is written in (1) and the corresponding SINR is defined as:

$$
\operatorname{SINR}_{k}^{D L}=\frac{p_{k} \mathbf{g}_{k}^{H} \mathbf{h}_{k k}^{H} \mathbf{h}_{k k} \mathbf{g}_{k}}{\sum_{l \neq k} p_{l} \mathbf{g}_{l}^{H} \mathbf{h}_{k l}^{H} \mathbf{h}_{k l} \mathbf{g}_{l}+\sigma^{2}}
$$

where $p_{k}$ is the TX power at the BS for the stream intended to the $k$-th user. Imposing a set of DL SINR constraints at each mobile station: $S I N R_{k}^{D L}=\gamma_{k}$ it is possible to rewrite equation (2) in matrix notation:

$$
\boldsymbol{\Phi} \mathbf{p}+\boldsymbol{\sigma}=\mathbf{D}^{-1} \mathbf{p}
$$

where the two matrices $\boldsymbol{\Phi}$ and $\mathbf{D}$ are defined in (4) and (5), $\mathbf{p}=\left[p_{1}, \ldots, p_{K}\right]^{T}$ and $\boldsymbol{\sigma}=\sigma^{2} \mathbf{1}$ are two vectors that contain all the TX powers and and the noise variances respectively. 1 is a column vector of dimensions $K \times 1$ that contains all ones.

$$
[\boldsymbol{\Phi}]_{i j}= \begin{cases}\mathbf{g}_{j}^{H} \mathbf{h}_{i j}^{H} \mathbf{h}_{i j} \mathbf{g}_{j}, & j \neq i \\ 0, & j=i\end{cases}
$$

$$
\mathbf{D}=\operatorname{diag}\left\{\frac{\gamma_{1}}{\mathbf{g}_{1}^{H} \mathbf{h}_{11}^{H} \mathbf{h}_{11} \mathbf{g}_{1}}, \ldots, \frac{\gamma_{K}}{\mathbf{g}_{K}^{H} \mathbf{h}_{K K}^{H} \mathbf{h}_{K K} \mathbf{g}_{K}}\right\}
$$

We can determine the TX power solving (3) w.r.t. $\mathbf{p}$ obtaining:

$$
\mathbf{p}=\left(\mathbf{D}^{-1}-\boldsymbol{\Phi}\right)^{-1} \boldsymbol{\sigma}
$$

Now we analyze the SINR in the SIMO UL IFC. Due to channel reciprocity we have that $\tilde{\mathbf{h}}_{k l}=\mathbf{h}_{l k}^{H} \forall k, l$ and the receiver filter in the UL is the reciprocal of the transmitter filter of the DL $\tilde{\mathbf{f}}_{k}=\mathbf{g}_{k}^{H}, \forall k$. The SINR for the UL channel can be written as:

$$
\operatorname{SINR}_{k}^{U L}=\frac{q_{k} \mathbf{g}_{k}^{H} \mathbf{h}_{k k}^{H} \mathbf{h}_{k k} \mathbf{g}_{k}}{\mathbf{g}_{k}^{H}\left(\sum_{l \neq k} q_{l} \mathbf{h}_{l k}^{H} \mathbf{h}_{l k}+\sigma^{2} \mathbf{I}\right) \mathbf{g}_{k}}
$$

where $q_{k}$ represent the Tx power from the $k$-th MS. Imposing a set of SINR constraints also in the UL: $S I N R_{k}^{U L}=\gamma_{k}$ it is possible to rewrite that constraints as:

$$
\tilde{\boldsymbol{\Phi}} \mathbf{q}+\boldsymbol{\sigma}=\mathbf{D}^{-1} \mathbf{q}
$$

where $\mathbf{D}$ is defined as in (5), $\mathbf{q}=\left[q_{1}, \ldots, q_{K}\right]^{T}$ and

$$
[\tilde{\boldsymbol{\Phi}}]_{i j}= \begin{cases}\mathbf{g}_{i}^{H} \mathbf{h}_{j i}^{H} \mathbf{h}_{j i} \mathbf{g}_{i}, & j \neq i \\ 0, & j=i\end{cases}
$$

the power vector can be found as:

$$
\mathbf{q}=\left(\mathbf{D}^{-1}-\tilde{\boldsymbol{\Phi}}\right)^{-1} \boldsymbol{\sigma}
$$

Comparing the definition in (4) and (9), we can see that $\tilde{\boldsymbol{\Phi}}=\boldsymbol{\Phi}^{T}$. This implies that there exists a duality relationship between the DL MISO and UL SIMO interference channels. To the best of our knowledge this is the first time that SINR duality has been addressed in the context of the IFC.

\section{MISO IFC VS. MISO BC}

It is also interesting to note that there is a strong parallel between the equations reported above to show the duality in the MISO interference channel and the ones used to prove duality in a BC-MAC in [2].

If we stack all the beamformers and the channel vectors in a matrix form, the cascade of channel and BF can be written as:

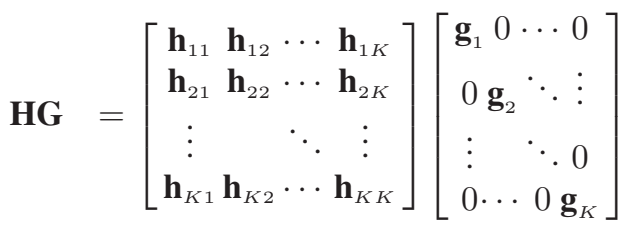

$$
\begin{aligned}
& =\left[\begin{array}{cccc}
\mathbf{h}_{11} \mathbf{g}_{1} & \mathbf{h}_{12} \mathbf{g}_{2} & \cdots & \mathbf{h}_{1 K} \mathbf{g}_{K} \\
\mathbf{h}_{21} \mathbf{g}_{1} & \mathbf{h}_{22} \mathbf{g}_{2} & & \vdots \\
\vdots & & \ddots & \mathbf{h}_{K-1 K} \mathbf{g}_{K} \\
\mathbf{h}_{K 1} \mathbf{g}_{1} & \cdots & \cdots & \mathbf{h}_{K K} \mathbf{g}_{K}
\end{array}\right]
\end{aligned}
$$

where the $\mathbf{G}$ is a block diagonal matrix and the diagonal blocks are BF column vectors. Making the notation in (11) more compact, denoting the $i$-th row of $\mathbf{H}$ as $\mathbf{H}_{i}$ and the $j$-th 
column of the BF matrix as $\mathbf{G}_{j}$ we have:

$$
\begin{aligned}
\mathbf{H G} & =\left[\begin{array}{c}
\mathbf{H}_{1} \\
\mathbf{H}_{2} \\
\vdots \\
\mathbf{H}_{K}
\end{array}\right]\left[\begin{array}{llll}
\mathbf{G}_{1} & \mathbf{G}_{2} & \cdots & \mathbf{G}_{K}
\end{array}\right] \\
& =\left[\begin{array}{cccc}
\mathbf{H}_{1} \mathbf{G}_{1} & \mathbf{H}_{1} \mathbf{G}_{2} & \cdots & \mathbf{H}_{1} \mathbf{G}_{K} \\
\mathbf{H}_{2} \mathbf{G}_{1} & \mathbf{H}_{2} \mathbf{G}_{2} & & \vdots \\
\vdots & & \ddots & \mathbf{H}_{K-1} \mathbf{G}_{K} \\
\mathbf{H}_{K} \mathbf{G}_{1} & \cdots & \cdots & \mathbf{H}_{K} \mathbf{G}_{K}
\end{array}\right]
\end{aligned}
$$

If we assume that the vector $\mathbf{H}_{i}$ represents the channel between the BS and the $i$-th MS and the vector $\mathbf{G}_{i}$ is the corresponding BF, equation (12) can be used to represent the $\mathrm{BC}$ channel. This makes the parallel between a BC and an interference channel more clear. In a similar fashion it is possible to describe the same parallelism between the $\mathrm{BC}$ and the interference channel for the UL SIMO IFC using similar matrix notation. Once we have shown this parallel between the two systems (BC and IFC) it is possible to extend the results obtained for the UL-DL duality in the BC-MAC to the IFC under a sum power constraint. A set of SINRs $\gamma_{1}, \ldots, \gamma_{K}$ is feasible whenever there exists a positive power allocation such that (3) for the DL ((8) for the UL) is fulfilled. In [2] the following is proved for the BC-MAC duality but it is also valid for the IFC: Targets $\gamma_{1}, \ldots, \gamma_{K}$ are jointly feasible in $U L$ and $D L$ if and only if the spectral radius $\rho$ of the weighted coupling matrix satisfies $\rho(\mathbf{D} \Phi)<1$.

Because $\rho(\mathbf{D} \Phi)=\rho\left(\mathbf{D} \Phi^{T}\right)$ target SINRs are feasible in the UL if and only if the same targets are feasible in the DL. The power allocation vectors that satisfy that constraints can be found using (6), for the DL, and (10), for the UL.

The total required UL power $q_{t o t}=\sum_{i} q_{i}$ is the same as the DL power $p_{\text {tot }}=\sum_{i} p_{i}$, this can be simply shown as follows:

$$
\begin{aligned}
\sum_{i} q_{i}= & \mathbf{1}^{T} \mathbf{q}=\sigma \mathbf{1}^{T}\left(\mathbf{D}^{-1}-\mathbf{\Phi}^{T}\right)^{-T} \\
& =\sigma \mathbf{1}^{T}\left(\mathbf{D}^{-1}-\mathbf{\Phi}\right)^{-1}=\sum_{i} p_{i}
\end{aligned}
$$

According to the relationship (13) it is possible to state that both UL and DL have the same SINR feasible region under a sum-power constraint, i.e., target SINRs are feasible in the DL if and only if the same targets are feasible in the UL. Using the results obtained before it is possible to extend some beamforming design techniques that use the BC-MAC duality to the beamforming design for a MISO IFC. In the following we report two problems in [3] and their application to the IFC.

\section{A. Max-Min SINR beamforming design (SINR Balancing)}

In many practical scenarios we have a $K$-user interference channel where each users must achieve individual target quality of service (QoS). The total transmission power available in the network (sum power at all Tx) is limited by $P_{t o t}$. We can define the following BF design problem:

$$
\begin{aligned}
\max & \min _{k: 1, \ldots, K} \frac{S I N R_{k}}{\gamma_{k}} \\
\text { s.t. } & \sum_{k} p_{k} \leq P_{t o t}
\end{aligned}
$$

\section{B. Power minimization under SINR constraints}

Another problem formulation of interest is to design the beamformers such that the total transmit power is minimized imposing a set of QoS constraints to each user. The problem now can be formulated as follows:

$$
\begin{array}{r}
\quad \min \sum_{k} p_{k} \\
\text { s.t. } \quad \frac{S I N R_{k}}{\gamma_{k}} \geq 1 \forall k
\end{array}
$$

The two problems can be efficiently solved using the iterative algorithm reported in [3] making the proper modification to adapt the algorithm to the interference channel.

\section{UL-DL DUALITY IN MISO/SIMO INTERFERENCE Channel Under Per User Power Constraint}

In the MISO interference channel if the problem of $\mathrm{BF}$ design is formulated under the sum power constraint we have shown that there exist an UL-DL duality in this kind of channels that can be used to solve the problem. Even though the sum power constraint is analytically attractive such constraint is not enough in a practical interference channel. In reality each user is subject to a per user power constraint that the transmit power can not violate. For this reason in this section we will introduce an alternative BF design that still minimize the total Tx power but imposing also per user power constraints. Here we will introduce a different UL-DL relation for the MISO IFC based on Lagrangian duality [7] that was previously introduced for the BC channel in [8]. For the rest of the paper we assume that the SINR constraints are such that there exist at least a feasible solution. The problem now becomes:

$$
\begin{aligned}
& \min _{\mathbf{g}_{1}, \ldots, \mathbf{g}_{K}, \alpha} \alpha \sum_{k=1}^{K} P_{k} \\
& \mathbf{g}_{k}^{H} \mathbf{g}_{k} \leq \alpha P_{k} ; k=1, \ldots, K \\
& \text { s.t. } \quad S I N R_{k}^{D L}=\frac{\mathbf{g}_{k}^{H} \mathbf{h}_{k k}^{H} \mathbf{h}_{k k} \mathbf{g}_{k}}{\sum_{l \neq k} \mathbf{g}_{l}^{H} \mathbf{h}_{k l}^{H} \mathbf{h}_{k l} \mathbf{g}_{l}+\sigma_{k}^{2}} \geq \gamma_{k} ; k=1, \ldots, K
\end{aligned}
$$

where $P_{k}$ represents the maximum Tx power for user $k$. The Lagrange dual of original DL problem in (16) can be stated as follows:

$$
\begin{gathered}
\max _{\substack{\lambda_{1}, \ldots, \lambda_{K}, \mu_{1}, \ldots, \mu_{K}, \\
\text { s.t. }}} \sum_{k=1}^{K} \lambda_{k} \sigma_{k}^{2} \\
\\
\sum_{k=1}^{K} P_{k}-\sum_{k=1}^{K} \mu_{k} P_{k} \geq 0 ; k=1, \ldots, K
\end{gathered}
$$

where $\lambda_{k}$ represents the Lagrange multiplier of the $k$-th SINR constraint and $\mu_{k}$ is the Lagrange multiplier associated to the Tx power constraint at user $\mathrm{k}$. Because strong duality holds between the original problem (16) and its dual (17) the optimal solution of the dual problem is also optimal for the original one. The proof that the duality gap is zero between the two optimization problems is not reported in this paper due to lack of space. It is essentially based on converting the non convex original problem into a convex problem.

The Lagrange dual of the DL beamforming problem (16) can be rewritten as an equivalent UL optimization problem for 
the Rx filter:

$$
\tilde{\mathbf{g}}_{k}=\left(\sum_{l \neq k} \lambda_{l} \mathbf{h}_{l k}^{H} \mathbf{h}_{l k}+\mu_{k} \mathbf{I}\right)^{-1} \mathbf{h}_{k k}^{H} \lambda_{k}
$$

in which the Tx power $\lambda_{k}$ and the noise power $\mu_{k}$ are to optimized. In the UL problem, in (19), each user transmits with power $\lambda_{k}, \forall k$, and the optimal value of the dual UL noise at the receiver is represented by $\mu_{k}, \forall k$ :

$$
\begin{gathered}
\max _{\lambda_{1}, \ldots, \lambda_{K}, \mu_{1}, \ldots, \mu_{K}} \sum_{k=1}^{K} \lambda_{k} \sigma_{k}^{2} \\
S I N R_{k}^{U L}=\frac{\lambda_{k} \tilde{\mathbf{g}}_{k}^{H} \mathbf{h}_{k k}^{H} \mathbf{h}_{k k} \tilde{\mathbf{g}}_{k}}{\tilde{\mathbf{g}}_{k}^{H}\left(\sum_{l \neq k} \lambda_{l} \mathbf{h}_{l k}^{H} \mathbf{h}_{l k}+\mu_{k} \mathbf{I}\right) \tilde{\mathbf{g}}_{k}} \leq \gamma_{k} ; k=1, \ldots, K \\
\sum_{k=1}^{K} P_{k}-\sum_{k=1}^{K} \mu_{k} P_{k} \geq 0 ; k=1, \ldots, K
\end{gathered}
$$

At the optimum the SINR constraints in the UL and the DL problems must be satisfied with equality. Using this relationship it is possible to derive the DL BF from the UL receiver filter. Because a scaling factor in the receiver filter at the BS does not affect the SINR it is possible to show that the optimal DL BFs are given by:

$$
\mathbf{g}_{k}=\sqrt{\beta_{k}} \tilde{\mathbf{g}}_{k}
$$

where $\beta$ is such that the SINRs in the DL are satisfied with equality so:

$$
\left[\begin{array}{c}
\boldsymbol{\beta}=\mathbf{D}^{-1} \boldsymbol{\sigma} \\
{[\mathbf{D}]_{i j}= \begin{cases}\frac{1}{\gamma_{i}} \tilde{\mathbf{g}}_{i}^{H} \mathbf{h}_{i i}^{H} \mathbf{h}_{i i} \tilde{\mathbf{g}}_{i} & i=j \\
-\tilde{\mathbf{g}}_{j}^{H} \mathbf{h}_{i j}^{H} \mathbf{h}_{i j} \mathbf{g}_{j} & i \neq j \\
-\end{cases} }
\end{array}\right.
$$

\section{Optimization In Presence of Primary Users}

In the $\mathrm{CR}$ scenario the $\mathrm{BF}$ design of the opportunistic users must take into account the presence of the primary nodes. In the underlay paradigm, the secondary users are allowed to transmit if the interference caused to the primary users is below a fixed threshold. In this section we will applied the beamforming techniques described previously in the paper to a CR setting, constraining the total interference power caused to the primary user due to opportunistic transmission to be limited. We assume that the secondary network is represented by a $K$-user MISO interference channel that communicate opportunistically in the presence of $L$ single antenna primary receivers.

\section{A. CR Beamformer Design Under Per User Power Constraint}

In a CR setting the BF design problem reported in (16) should be modified introducing additional inequality constraints to control the total interference caused by the SN Tx at the individual PUs:

$$
\begin{gathered}
\min _{1} \alpha \sum_{k=1}^{K} P_{k} \\
\mathbf{g}_{k}^{H} \mathbf{g}_{k} \leq \alpha P_{k} ; k=1, \ldots, K \\
\text { s.t. } \quad S I N R_{k}^{D L}=\frac{\mathbf{g}_{k}^{H} \mathbf{h}_{k k}^{H} \mathbf{h}_{k k} \mathbf{g}_{k}}{\sum_{l \neq k} \mathbf{g}_{l}^{H} \mathbf{h}_{k l}^{H} \mathbf{h}_{k l} \mathbf{g}_{l}+\sigma_{k}^{2}} \geq \gamma_{k} ; k=1, \ldots, K \\
\frac{1}{I_{m}^{D L}}=\frac{1}{\sum_{k=1}^{K} \mathbf{g}_{k}^{H} \mathbf{h}_{K+m k}^{H} \mathbf{h}_{K+m k} \mathbf{g}_{k}} \geq \gamma_{K+m} ; m=1, \ldots, L
\end{gathered}
$$

where $\mathbf{h}_{K+m k}$ is the downlink channel between the $k$-th transmitter and the $m$-th PU and $\gamma_{K+m}$ is a measure related to the interference level $I_{m}$ at $m$ th PU.
The augmented problem can now be treated as additional virtual SISO Tx/Rx pairs in the IFC. This nevertheless does not change the UL-DL duality if one considers further (fictitious) interferers in the SN transmitting powers [9], $p_{m}, m=K+$ $1, \ldots, K+L$ to the corresponding $\mathrm{PU}$ while causing zero interference to the $K$ links (receivers) of the SN. Introducing this modification the interference constraint in (22) can be rewritten as:

$$
\frac{1}{I_{m}^{D L}}=\frac{p_{K+m}}{\sum_{k=1}^{K} \mathbf{g}_{k}^{H} \mathbf{h}_{K+m k}^{H} \mathbf{h}_{K+m k} \mathbf{g}_{k}} \geq \gamma_{K+m} ; m=1, \ldots, L
$$

From the augmented $\mathrm{BF}$ design problem it is possible to derive the Lagrange dual problem:

$$
\begin{array}{ll} 
& \min _{\lambda_{k}, \mu_{k}, p_{K+m},} \sum_{k=1}^{K} \lambda_{k} \sigma_{k}^{2}-\sum_{m=1}^{L} \frac{\lambda_{K+l} p_{K+m}}{\gamma_{K+m}} \\
\text { s.t. } \quad- & \frac{\lambda_{k}}{\gamma_{k}} \mathbf{h}_{k k}^{H} \mathbf{h}_{k k}+\sum_{l \neq k}^{K+L} \lambda_{l} \mathbf{h}_{l k}^{H} \mathbf{h}_{l k}+\mu_{k} \mathbf{I} \succeq 0 ; k=1, \ldots, K \\
& \sum_{k=1}^{K} P_{k}-\sum_{k=1}^{K} \mu_{k} P_{k} \geq 0 \quad k=1, \ldots, K
\end{array}
$$

The Lagrange dual can be interpreted as a dual UL problem as done in section V where the LMI constraint can be seen as a dual UL SINR constraint in which there are some fictitious link between the PU nodes and the SU receivers. As we can see introducing the per user power constraint brings to introduce an uncertain noise in the dual problem that should also be determined.

\section{B. CR Beamformer Design Under Sum Power Constraint}

We stick with the original $\mathrm{BC}$ objective of designing the $\mathrm{BF}$ to minimize the total transmitted power subject to some minimum QoS requirements for each user constraining also the total interference caused by the SN Tx at the individual PUs. This problem can be expressed as

$$
\begin{aligned}
& \min _{\mathbf{g}_{1}, \ldots, \mathbf{g}_{K}} \sum_{k=1}^{K} \mathbf{g}_{k}^{H} \mathbf{g}_{k} \\
& \begin{array}{l}
\text { s.t. } \quad S I N R_{k}^{D L}=\frac{\mathbf{g}_{k}^{H} \mathbf{h}_{k k}^{H} \mathbf{h}_{k k} \mathbf{g}_{k}}{\sum_{l \neq k} \mathbf{g}_{l}^{H} \mathbf{h}_{k l}^{H} \mathbf{h}_{k l} \mathbf{g}_{l}+\sigma_{k}^{2}} \geq \gamma_{k} ; k=1, \ldots, K \\
\frac{1}{I_{m}^{D L}}=\frac{1, \ldots, L}{\sum_{k=1}^{K} \mathbf{g}_{k}^{H} \mathbf{h}_{K+m k}^{H} \mathbf{h}_{K+m k} \mathbf{g}_{k}} \geq \gamma_{K+m} ; m=1, \ldots, L
\end{array}
\end{aligned}
$$

The BF design addressed here was studied for the CR BC case in [4], and in [9]. Here we extend their work to the MISO IFC.

The constrained problem can be directly solved using a technique proposed in [4]. Alternatively, we consider here the extended downlink IFC and introducing the fictitious link transmit powers $p_{K+m}$ [9]. Using this method the interference constraint in (25) can be rewritten as in (23).

The resulting $K+L$ user augmented IFC can be solved for beamformers and power allocations using the duality principle as discussed in the previous sections.

\section{CR BF Design ALGORIThm}

In this section we report two numerical algorithms to solve the problem of optimal downlink beamformer design with per user power constraints in a CR setting as stated in section VI-A. The Euclidean projection $\mathcal{P}_{S_{\mu}}$ in (26) is done on the 


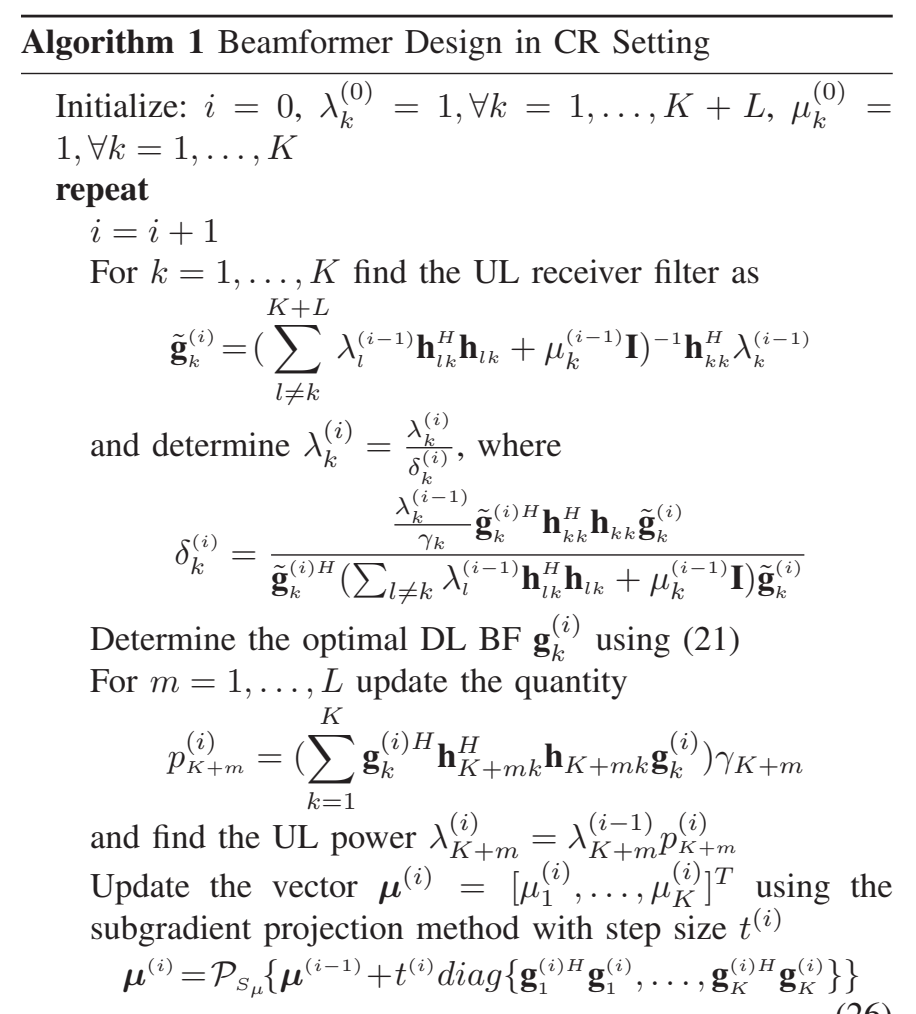

until convergence

constraint set $S_{\mu}=\left\{\boldsymbol{\mu}: \sum_{k=1}^{K} P_{k}-\sum_{k=1}^{K} \mu_{k} P_{k}, \mu_{k} \geq 0\right\}$. The first algorithm, Table 1 , is an iterative algorithm that solves the Lagrange dual problem (24). This algorithm also allows to solve the problem with sum power constraint by simply removing the subgradient projection operation is (26) The second algorithm that we propose solves the Lagrange dual problem (24) using the interior-point-method where the LMI constraint, that represents the dual UL SINR is handled using a logarithmic barrier [7]. In Fig. 2 is plotted the Normalized Root Mean Square Error (NRMSE),

$$
N R M S E=\frac{\sqrt{\frac{1}{N} \sum_{n=1}^{N} \sum_{k=1}^{K}\left(\left\|\mathbf{g}_{k}^{(i)}(n)\right\|_{2}-\left\|\mathbf{g}_{k}^{*}(n)\right\|_{2}\right)^{2}}}{\sqrt{\frac{1}{N} \sum_{n=1}^{N} \sum_{k=1}^{K}\left(\left\|\mathbf{g}_{k}^{*}(n)\right\|_{2}\right)^{2}}}
$$

where $\left\|\mathbf{g}_{k}^{(i)}(n)\right\|_{2}$ represents the Euclidean norm of the DL BF determined using the iterative algorithm at iteration $(i)$ for the $n$-th Monte Carlo run and $\mathbf{g}_{k}^{*}(n)$ is the DL BF obtained using the interior point method. The considered system is given by a secondary IFC of $K=5$ users with $M=9$ Tx antennas each and $L=5$ PU. The target SINR are $\gamma_{k}=1$ for all SU and the interference constraints are $\gamma_{K+m}=, \forall m$, and the noise variance is equal to $-10 d B$.

\section{CONCLUSIONS}

Uplink-downlink duality holds in the IFC in the form of a MISO-SIMO SINR duality. In particular the dual of an IFC is still an IFC, the advantage of duality is that the beamformer design problem is simplified in the dual UL SIMO IFC. We

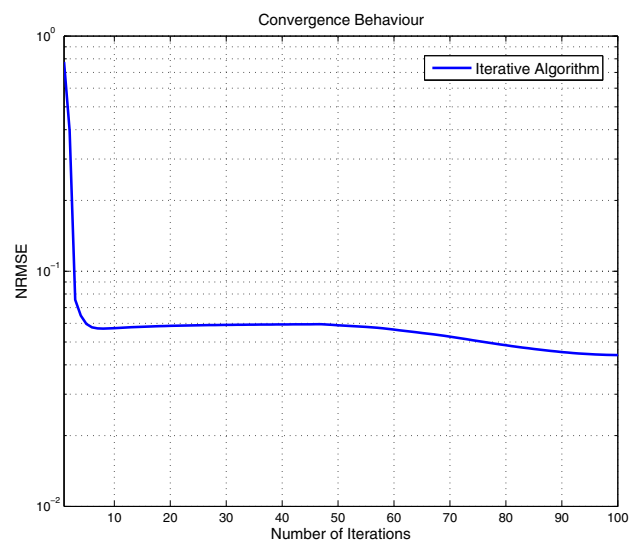

Fig. 2: NRMSE for $K=5, L=5, M=9$

also show that the underlying mechanics of this duality regime are similar to the UL-DL duality in the BC setting. This observation allows beamformer design in the MISO IFC using the same techniques as the ones well-known in the $\mathrm{BC}$ channel.

Beamformer design in presence of primary interference constraints is addressed. The primary users can be seen as a pairs of receiver and virtual primary Tx, thus supplementary interference links but causing zero interference to the SN. The extended problem can be readily solved using the new result on UL-DL IFC duality obtained in this paper.

\section{ACKNOWLEDGMENT}

EURECOM's research is partially supported by its industrial members: BMW Group Research \& Technology, Swisscom, Cisco, ORANGE, SFR, Sharp, ST Ericsson, Thales, Symantec, Monaco Telecom and by the EU FP7 NoE Newcom++.

The research of EURECOM and Infineon Technologies France is also supported in part by the EU FP7 Future and Emerging Technologies (FET) project CROWN.

\section{REFERENCES}

[1] Rui Zhang and Ying-Chang Liang, "Exploiting multi-antennas for opportunistic spectrum sharing in cognitive radio networks," Selected Topics in Signal Processing, IEEE Journal of, vol. 2, no. 1, pp. 88 -102, feb. 2008.

[2] H. Boche and M. Schubert, "A general duality theory for uplink and downlink beamforming," 2002, vol. 1, pp. 87 - 91 vol.1.

[3] M. Schubert and H. Boche, "Solution of the multiuser downlink beamforming problem with individual SINR constraints," Vehicular Technology, IEEE Transactions on, vol. 53, no. 1, pp. 18 - 28, jan. 2004

[4] K. Cumanan, L. Musavian, S. Lambotharan, and A.B. Gershman, "SINR balancing technique for downlink beamforming in cognitive radio networks," Signal Processing Letters, IEEE, vol. 17, no. 2, pp. $133-136$, feb. 2010.

[5] K. Gomadam, V.R. Cadambe, and S.A. Jafar, "Approaching the capacity of wireless networks through distributed interference alignment," in Proc. IEEE Global Telecommunications Conf. (GLOBECOM), Dec 2008.

[6] F. Negro, S. Shenoy, D.T.M. Slock, and I. Ghauri, "Interference alignment limits for $K$-user frequency-flat MIMO interference channels," in Proc. European Signal Proc. Conf. (Eusipco), Glasgow, Scotland, Aug. 2009.

[7] S. Boyd and L. Vandenberghe, Eds., Convex Optimization, Cambridge Univ. Press,, Cambridge U.K., 2004.

[8] W. Yu and T. Lan, "Transmitter optimization for the multi-antenna downlink with per-antenna power constraints," IEEE Trans. on Signal Processing, vol. 55, no. 6, June 2007.

[9] M. Pesavento, D. Ciochina, and A.B. Gershman, "Iterative dual downlink beamforming for cognitive radio networks,," submitted to CROWNCOM 2010, June 2010 . 